\title{
MEDICIÓN, RENDIMIENTO Y CALIDAD. LAS POLÍTICAS DE EVALUACIÓN DEL SISTEMA EDUCATIVO EN LA ARGENTINA RECIENTE (2015-2019)*
}

Lucrecia Rodrigo

\section{Introducción}

En Argentina la llegada del gobierno de la Alianza Cambiemos al poder, a fines del 2015, supuso una transformación en las orientaciones de las políticas públicas. En el campo escolar, a partir del diagnóstico de crisis del sistema educativo, comienza a delinearse una nueva agenda donde los debates en torno a la calidad se vinculan a la medición estandarizada de los resultados escolares y al modelo de las competencias que el mundo económico y global requiere, tomándose como referencia las pruebas PISA de la OCDE. En este contexto, las estrategias de evaluación de los procesos educativos bajo la lógica de la rendición de cuentas y la responsabilidad por los resultados, se convierten en ejes claves y transversales de la política educativa orientada a la mejora de la calidad. A partir de la identificación de las principales iniciativas llevadas adelante por el Ministerio de Educación de la Nación en el área de la evaluación, durante el período 2015-2019, el artículo se propone analizar las orientaciones que asumen dichas políticas en el país. Para tal fin, en primer lugar, se presenta un breve recorrido por las políticas de evaluación desde los años 1990 cuando se instalan como tema de agenda. En segundo término, se describen las principales medidas de evaluación impulsadas por el Ministerio de Educación nacional durante los años en cuestión. Finalmente, en tercer lugar, se recuperan algunas discusiones conceptuales sobre los sentidos de la evaluación bajo el propósito de analizar, para el caso argentino, los significados que se han construido en torno a la evaluación como dispositivo pedagógico privilegiado para alcanzar la calidad.

La evaluación de la calidad educativa es una tendencia internacional que se ha instalado con fuerza como política pública en el mundo globalizado. Bajo la lógica de la rendición de cuentas y la responsabilidad por los resultados (Falabella, 2015), desde los años 1980 distintos países han creado o fortalecido agencias gubernamentales destinadas a la evaluación de sus sistemas educativos (Ravela et al., 2008; Ferrer, 2006; Toranzos, 2014). Países como Argentina no han sido ajenos al ímpetu evaluador. En la Ley Federal de Educación de 1993 (LFE N²4.195) se dispuso que el Ministerio de Educación de la Nación, en coordinación con las jurisdicciones, evaluara de manera permanente la calidad del sistema educativo. De allí, la creación del Sistema Nacional de Evaluación en 1995 y la consecuente aplicación de las pruebas nacionales de aprendizaje escolar. En ese decenio se alentó también la participación en experiencias globales de evaluación, que tomaron impulso en años posteriores de la mano de organizaciones internacionales como el Banco Mundial, la Unesco y la OCDE.

${ }^{*}$ DOI - 10.29388/978-65-86678-87-1-0.f.15-27 
Si durante los años 1990, en el marco de las reformas educativas neoliberales, la evaluación quedó vinculada a la preocupación por la calidad en términos de eficacia del sistema educativo, la llegada del nuevo siglo supuso en el país perspectivas renovadas que buscaron reivindicar la calidad como un derecho (Pascual y Albergucci, 2016). A partir de entonces, la política educativa nacional quedó orientada por medidas tendientes a la búsqueda de igualdad e inclusión (Feldfeber y Gluz, 2011). Estas iniciativas se expresaron en la sanción de nuevas leyes, entre las cuales cabe destacar la Ley de Educación Nacional (LEN N²6 206) de 2006, que estableció el acceso universal y obligatorio al nivel secundario y posicionó al Estado como garante de este proceso. La ley definió un nuevo marco regulatorio para la evaluación, que fue concebida como uno de los instrumentos destinados a alcanzar la igualdad y calidad, extendiendo su mirada no solo a los resultados en las pruebas, sino también al contexto y a las condiciones de producción de la enseñanza y el aprendizaje.

A partir del 2015, con la llegada del gobierno nacional de la Alianza Cambiemos, nuevas orientaciones marcan el rumbo de la agenda educativa y aunque la política de evaluación desplegada desde entonces continuó regulada por la LEN, comenzaron a diseñarse propuestas que revalorizaron a la evaluación como uno de los medios privilegiados para mejorar la calidad. ${ }^{1}$ En este contexto, la información sobre la calidad volvió a equipararse a los resultados en pruebas estandarizadas y a gran escala, acercándose a los sentidos tecnocráticos que predominaron durante los años 1990.

En un escenario nacional e internacional de profundización de las orientaciones hacia la estandarización y medición de los procesos educativos, el artículo identifica y discute las tendencias que asumieron las políticas destinadas a la evaluación bajo el gobierno de la Alianza Cambiemos (2015-2019). ${ }^{2}$ Para tal fin, la exposición se organiza en tres apartados. En primer lugar, se presenta un breve recorrido histórico por las políticas de evaluación en el país desde los años 1990, cuando se instalan como tema de agenda y se asocian a la eficacia escolar, pasando por los gobiernos de principio de siglo XXI que resignifican y amplían sus sentidos hasta fines del año 2015, cuando se retoman concepciones asociadas a la medición y se revaloriza su función social. En segundo lugar, se presentan las principales intervenciones de evaluación del sistema educativo impulsadas por el Ministerio de Educación de la Nación durante el gobierno de Cambiemos que, en su conjunto, expresan la revalorización de estas acciones en términos de medición de la calidad. Finalmente, se recuperan algunas discusiones conceptuales sobre los sentidos de la evaluación como mecanismo de regulación y control de las prácticas educativas durante la gestión bajo estudio.

\section{Un breve recorrido por las políticas de evaluación en Argentina}

\footnotetext{
${ }^{1}$ El gobierno de la Alianza Cambiemos, una coalición política nacional, ganó las elecciones presidenciales en Diciembre de 2015. Mauricio Macri fue elegido entonces presidente del país.

2 Este artículo se desprende del proyecto UBACyT "Políticas públicas y derecho a la educación en la Argentina del siglo XXI. Análisis sobre las trayectorias de las políticas educativas en un país federal", radicado en el Instituto de Investigación en Ciencias de la Educación de la Universidad de Buenos Aires.
} 
En Argentina fue a partir de los años 1990 cuando la evaluación de la calidad, en términos de eficacia del sistema educativo, se instala como tema de agenda. En este escenario, la LFE dispuso que el Ministerio de Educación de la Nación en coordinación con las provincias evaluara de manera permanente la calidad de la educación. Bajo este marco legal, se creó el Sistema Nacional de Evaluación de la Calidad Educativa en 1995 y comenzaron a aplicarse los Operativos Nacionales de Evaluación (Narodowsky, et al., 2002; Gvirtz, et al., 2006). A su vez, el país empezó a integrar, de manera sistemática, experiencias mundiales de evaluación, a tono con las recomendaciones de organismos internacionales como el Banco Mundial. Los datos proporcionados por estas pruebas se consideraron un indicador privilegiado del desempeño de los profesores, estableciéndose una relación causal entre los docentes que enseñan y los estudiantes que aprenden que condujo a plantear el "déficit" en la formación docente (Feeney y Diker, 1998).

En el país, igual que sucedió en buena parte de la región, la llegada del nuevo siglo dio lugar a la promoción de discursos que vincularon la calidad con una perspectiva de derechos. A partir de entonces, y bajo los denominados gobiernos kirchneristas (2003-2007; 2007-2015), la inclusión con calidad se convirtió en uno de los ejes nodales de la política educativa argentina de la época (Brener y Galli, 2016). ${ }^{3}$ Desde el discurso oficial se consideró a la inclusión escolar como un acto de justicia social y a la calidad como el resultado de acciones integrales orientadas a garantizar las condiciones materiales de la educación y la mejora de los procesos de enseñanzaaprendizaje (Feldfeber y Gluz, 2019). Resultado de esta manera amplia y compleja de comprender la calidad, la evaluación se alejó de interpretaciones reduccionistas que la limitaban a la medición del rendimiento y a la rendición de cuentas, para acercarla a visiones que destacaron sus aspectos formativos y menos técnicos, tal como quedó expresado en la LEN. En este nuevo marco legal, las políticas de evaluación extendieron su mirada no solo a los resultados, sino también al contexto y a las condiciones en que se producen los procesos de enseñanza y aprendizaje (Pascual y Albergucci, 2016). De allí, que se alentara la implementación de investigaciones y evaluaciones alternativas para efectuar diagnósticos institucionales.

Esta manera más amplia e integral de concebir la evaluación estuvo acompañada, sin embargo, por los tradicionales ONE que no lograron reconceptualizar sus sentidos y quedaron limitados a la medición del aprendizaje a través de pruebas estandarizadas y a gran escala. Algo similar sucedió con los estudios internacionales que el país continuó integrando, entre los cuales destacan las pruebas PISA de la OCDE. Si bien estas experiencias, ya institucionalizadas, siguieron funcionando, fueron también objeto de críticas y resistencias por parte de altos funcionarios del propio ministerio. En lo que respecta a las pruebas PISA, en particular, se cuestionó la escasa injerencia de los países periféricos en su diseño, ejecución e interpretación de resultados. Al mismo tiempo, se las consideró descontextualizadas de las realidades socioeconómicas de los estados latinoamericanos (Rodrigo, 2016 y 2019). En suma, y pese a los re-

\footnotetext{
${ }^{3}$ El kirchnerismo gobernó el país entre los años 2003 y 2015. La primera presidencia la ocupó Néstor Kirchner (2003-2007). El segundo y tercer gobierno kirchnerista, estuvo bajo la presidencia de Cristina Fernández de Kirchner (2007-2015).
} 
paros señalados, en el marco de la LEN y durante los tres gobiernos kirchneristas, los sentidos adoptados por la evaluación se ampliaron en paralelo a la redefinición de la noción de calidad.

\section{Reeditando la "cultura de la evaluación": las políticas del gobierno de la Alianza Cambiemos}

En 2005, la llegada al gobierno nacional de la Alianza Cambiemos inauguró una nueva coyuntura histórica para el país. Rápidamente, el nuevo gobierno procedió al ajuste económico y al progresivo desmantelamiento de las políticas sociales con importantes repercusiones en el campo escolar (Gluz, 2019; Feldfeber y Gluz, 2019). La agenda educativa fue reorientada y se renovaron temas y propuestas que, en buena medida, habían marcado el rumbo de la política educativa de los años 1990. Al respecto, las medidas y acciones orientadas a la evaluación de la calidad se convirtieron en uno de los ejes claves y transversales que articularon a la política educativa.

En sintonía con las recomendaciones de organismos internacionales como el Banco Mundial y la OCDE (Jakobi y Martens, 2007; Verger y Bonal, 2011), la política de evaluación quedó reducida a la aplicación de nuevas pruebas estandarizadas y a gran escala, consideradas el medio privilegiado para contar con datos válidos y objetivos sobre el estado de la educación. En esta línea, se cuestionó el valor de la información obtenida por los anteriores ONE y la manera "desinteresada" que distinguió a la participación del país en experiencias internacionales como PISA. En este contexto, se consideró fundamental contar con vehículos de recolección de información "transparentes" y "fiables" que se tradujeron en la aplicación de renovados dispositivos de evaluación.

Bajo estas redefiniciones, la política de evaluación adquirió un nuevo rumbo que se expresó en el diseño y ejecución de una serie de iniciativas y programas: 1) la creación de la Secretaría de Evaluación, 2) la puesta en marcha del Plan Nacional de Evaluación de la Calidad y Equidad y 3) la relevancia otorgada a la publicidad de los resultados de las evaluaciones.

\section{1- La Secretaría de Evaluación Educativa}

Una de las primeras propuestas de los representantes del Ministerio de Educación argentino, fue la creación del Instituto Nacional de Evaluación de la Calidad y Equidad Educativa. Este instituto pretendía convertirse en un organismo autárquico donde las decisiones y el financiamiento estarían fuera de la órbita del Ministerio. La creación del instituto no prosperó, pero por decreto se puso en marcha en el año 2016 la Secretaría de Evaluación Educativa dependiente del Ministerio de Educación. ${ }^{4}$

\footnotetext{
4 La Secretaría de Evaluación se creó en abril del año 2016 por medio del Decreto $\mathrm{N}^{\circ} 552$ del Poder Ejecutivo Nacional. Uno de sus principales antecedente fue la "Unidad de Evaluación Integral de la Calidad y Equidad Educativa" de la Ciudad Autónoma de Buenos Aires (CABA), creada como una entidad descentralizada con personería jurídica en el ámbito del Ministerio de Educación.
} 
La justificación de la nueva institucionalidad radicó en la necesidad de fortalecer la eficacia de aquellas áreas dedicadas a la evaluación e información del sistema, para lo cual se consideró dar lugar a una nueva agencia con facultades exclusivas sobre la evaluación. Específicamente, la Secretaría se propuso como el ámbito privilegiado para la generación, coordinación y concertación federal del nuevo sistema de evaluación. Para tal fin, se buscó examinar tres aspectos claves del funcionamiento del sistema: 1) el desempeño de los estudiantes y las condiciones bajo las cuales se desarrolla, diseñando para tal fin el dispositivo nacional Aprender que reemplazó a los anteriores ONE; 2 ) las políticas públicas en materia de educación, su funcionamiento e impacto y 3) las instituciones educativas a través de la Autoevaluación Institucional Aprender, que se presentó como una modalidad participativa, democrática y formativa, ya presente en la LEN, cuyo objetivo consistía em que los propios actores identifiquen y propongan acciones para la mejora del proyecto institucional.

Los tres aspectos a evaluar se tradujeron en objetivos específicos de la Secretaría, entre los cuales destacan: fortalecer la capacidad de análisis de los actores del sistema; promover el uso de la información para la toma de decisiones; diagnosticar problemas, monitorear condiciones y resultados educativos; definir estrategias de gestión y de acción; entre los más relevantes. En su conjunto, estos propósitos dieron lugar a "la implementación de un sistema de información y evaluación de la educación articulado, confiable, pertinente, amigable, dinámico y periódico que sirva a los distintos usuarios del sistema educativo y promueva el uso de evidencia para la toma de decisiones en pos de incidir en la mejora continua de la educación y en la disminución de las brechas de desigualdad", tal como quedó establecido en su decreto de creación (Decreto PE $N^{\circ}$ 552/16). La nueva Secretaría se propuso así, fortalecer la cultura de la evaluación, desarrollando e implementando una estrategia de generación de conocimiento centrada en ciertas problemáticas educativas, bajo el fin de proveer evidencia para la gestión y la comunidad en su conjunto. Cabe señalar que las acciones de la Secretaría debían ser acordadas en el ámbito del Consejo Federal de Educación (CFE). Dado que las decisiones tomadas en el seno de la Asamblea del CFE tienen carácter obligatorio para las provincias, este organismo es quien debe garantizar la participación y articulación entre las jurisdicciones del país.

\subsection{El Sistema de Evaluación Nacional de la Calidad y Equidad Educativa: los programas nacionales, internacionales y la publicación de sus resultados}

Bajo la nueva arquitectura institucional que supuso la creación de la Secretaría de Evaluación, en 2016 el CFE aprueba una nueva resolución que establece la implementación y los lineamientos generales del Sistema de Evaluación Nacional de la Calidad y Equidad Educativa, cuya coordinación y seguimiento recae en la Secretaría (Res. CFE N²80/16). Como se estableció en la normativa de creación, este sistema se organizó en torno a cuatro áreas. La primera, referida al diseño y aplicación de dispositivos nacionales, regionales e internacionales de evaluación. La segunda, vinculada a los procesos de autoevaluación institucional en los niveles obligatorios. La 
tercera, relacionada con la evaluación de programas y proyectos por parte de los gobiernos educativos. La cuarta, asociada al fortalecimiento federal de las capacidades estatales en materia de evaluación a partir de la conformación de una Red de Evaluación Federal de Calidad y Equidad Educativa (REFCEE). ${ }^{5} \mathrm{~A}$ través de esta red se propuso aunar esfuerzos entre los distintos niveles de gobierno (nacional y jurisdiccional) para fortalecer la denominada cultura de la evaluación. ${ }^{6}$

Dentro de este esquema nacional, el programa Aprender se propuso como el instrumento privilegiado de la Secretaría destinado a revitalizar los operativos de evaluación. Fue a través de este programa que se pretendió concientizar a la sociedad sobre la relevancia de las evaluaciones nacionales como instancias de aprendizaje y transparencia sobre el estado de la educación. ${ }^{7}$ De hecho, su objetivo fue obtener periódicamente información para el análisis y la toma de decisiones acerca de los desempeños de los estudiantes en los niveles primario y secundario. En un comienzo se decidió que las pruebas se aplicarían de manera anual, obligatoria y censal, tanto para los estudiantes de sexto grado de primaria como para los de quinto/sexto año del secundario. Las áreas establecidas a evaluar fueron Matemática, Lengua, Ciencias Naturales, y Sociales. ${ }^{8}$ A su vez, junto a las pruebas, el programa se distingue por aplicar cuestionarios complementarios que relevan opiniones de los estudiantes y equipos directivos acerca de las condiciones en las que se desenvuelve el aprendizaje escolar. Desde el año 2018, las pruebas Aprender ya no se aplican anualmente en los dos niveles, sino que en cada año se privilegia la evaluación de un nivel educativo en particular. Los motivos que impulsaron dicha decisión fueron, sobre todo, de índole económica, dado el significativo recorte que sufrió el sector durante los últimos presupuestos anuales bajo la gestión del gobierno de Cambiemos. ${ }^{9}$

Junto a las pruebas Aprender, se avanzó también en el diseño de propuestas de evaluación del sistema de formación docente. En 2017 se implementó el programa Enseñar que se presentó como una evaluación diagnóstica de carácter nacional de los

\footnotetext{
${ }^{5}$ La REFCEE está integrada por las Unidades Jurisdiccionales de Evaluación que cada provincia implementó en el curso del primer semestre de 2016.

${ }^{6}$ Con ello se da cumplimiento a la LEN, que establece que las provincias, la Ciudad Autónoma de Buenos Aires y la Nación participarán del sistema de evaluación de la educación, y garantizarán su implementación conforme a sus respectivas responsabilidades.

${ }^{7}$ Específicamente, se establece que Aprender es el dispositivo nacional de evaluación de los aprendizajes e información sobre las condiciones en las que éstos se desarrollan que cumple con lo dispuesto por la LEN. Los contenidos y su forma de implementación fueron acordados en el marco del CFE (Res. N²80/16).

${ }^{8}$ La primera evaluación Aprender fue en el año 2016 y se evaluó de manera censal a los estudiantes de sexto año de primaria en lengua y matemática. Se aplicó también pruebas em quinto/sexto año del secundario en lengua, matemática, ciencias sociales y ciencias naturales. En esta primera edición, se examinó a su vez una muestra de tercero de primaria y de segundo/tercer año de secundaria en lengua y matemática. Aprender 2017 también aplicó pruebas de manera censal en sexto año de primaria en ciencias sociales y naturales, y en quinto/sexto de secundaria en lengua y matemática. A una muestra de cuarto año de primaria se evaluó durante esta edición en producción escrita. Durante el año 2018 se examinó de manera censal a los estudiantes de sexto año de primaria en lengua y matemática; mientras que durante el mes de Septiembre del año 2019 se evaluó a todos los alumnos de quinto/sexto de secundaria en las mismas áreas. En esta edición se aplicó también pruebas a una muestra de estudiantes en las áreas de ciudadanía y ciencias naturales; evaluando, a su vez, una muestra de tercer año en aprendizaje digital.

9 Al respecto, ver el informe de los presupuestos educativos del Estado Nacional para el período de gestión de gobierno 2016-2019. Disponible en: https://drive.google.com/file/d/10kW1xvcdy09sP5h_LdeJVTWy-ThzZMT6/ view.
} 
estudiantes del último año de las carreras de formación docente de Educación Primaria y de asignaturas del Ciclo Básico de la Educación Secundaria. Aunque este programa sólo se editó por única vez, puso en evidencia la centralidad de la medición del desempeño de los docentes en la agenda educativa.

Finalmente, adquieren centralidad en el plan nacional de evaluación los operativos internacionales y regionales impulsados por organismos internacionales con injerencia en el campo escolar. Al respecto, destacan las pruebas PISA de la OCDE y las evaluaciones del Laboratorio Latinoamericano de la Evaluación de la Calidad Educativa (LLECE) de la Unesco, que fueron consideradas por la gestión de Cambiemos parámetros privilegiados del estado de la calidad a nivel internacional.

En este contexto de revitalización de la cultura de la evaluación, la difusión de los resultados adquiere relevancia. De allí que, junto al programa Aprender, se ponga en funcionamiento el llamado Sistema Abierto de Consulta Aprender, orientado a que todos los "usuarios" de la educación accedan al procesamiento en línea de las bases de datos de las evaluaciones. A través de sus publicaciones, la plataforma se presentó como la instancia privilegiada para acceder a la información estadística, no sólo por parte de los organismos estatales sino también por la sociedad civil en su conjunto. La plataforma expresó la centralidad de la política de datos e información "transparente", que se convirtió en uno de los slogans de la época. De hecho, han sido frecuentes las declaraciones de distintos funcionarios orientadas a impulsar la publicación de los resultados de las pruebas Aprender por escuela, en tanto medio para mejorar la calidad. Vale señalar que en el país este es un tema sensible y polémico, pues entra en tensión con la prohibición de publicar los datos de las instituciones en las pruebas de rendimiento, tal como lo establece el artículo 97 de la LEN, que afecta a la publicación de información concerniente a investigaciones o evaluaciones educativas, y la ley nacional sobre el sistema estadístico ( $N^{\circ} 17.622$ ).

\section{Medición, estandarización y regulación: discutiendo las orientaciones de la política de evaluación bajo el gobierno de la Alianza Cambiemos}

A continuación, se recuperan algunas discusiones conceptuales sobre los sentidos de la evaluación bajo el gobierno argentino de Cambiemos. Para tal fin se identifican, en primer lugar, cuatro cuestiones que atraviesan al conjunto de acciones que integraron el plan nacional de evaluación y que ponen en evidencia lógicas tecnocráticas típicas de los años 1990 pero con especificidades propias de dicha gestión.

La primera, refiere a la reducción de la calidad a los resultados conseguidos en pruebas estandarizadas y a gran escala de logro escolar. Es a través de estas pruebas que pareciera posible contar con datos objetivos y fiables sobre el estado de la educación argentina. Se acepta, de este modo, que los resultados de las pruebas son evidencia suficiente para dar cuenta de los problemas educativos del país. Así, aunque estas evaluaciones constituyan un recorte de realidades escolares complejas, se afirma que sus datos conducen a una toma de decisiones informada. Las pruebas 
aparecen como los ejes del monitoreo de la educación, donde la calidad se limita a la medición del aprendizaje en ciertas áreas de conocimiento consideradas "claves" para alcanzar niveles aceptables de desarrollo. En este esquema, cabe agregar, las ciencias sociales vienen perdiendo terreno progresivamente.

En segundo lugar, investigaciones recientes han puesto en evidencia la relevancia de los datos de las pruebas Aprender como insumo clave en la construcción de políticas públicas específicas. En particular, en relación a la delimitación de las escuelas y sujetos destinatarios de programas estatales destinados a la intervención sobre las desigualdades socioeducativas. Se advierte así, que criterios meritocráticos e individualizantes inherentes al diseño de este tipo de pruebas, participan en la restauración de las fronteras de intervención estatal entre los distintos grupos sociales y en las maneras de concebir el derecho a la educación en políticas que adquieren rasgos de tipo focalizado (Gluz, 2019).

El tercer aspecto a destacar, se vincula con las propuestas de evaluación de los docentes. Estas estrategias, que han tomado impulso no sólo a nivel nacional sino también regional, se inscriben dentro de un escenario político de sospecha y desconfianza hacia el trabajo de los profesores y sus escuelas, tal como ponen en evidencia organizaciones internacionales como el Banco Mundial (2014) o el Programa Enseña por Argentina (Friedrich, 2014). Por otra parte, suelen orientarse al establecimiento de asociaciones entre el nivel de rendimiento docente y los incentivos económicos, cuestionando y poniendo en riesgo la estabilidad que históricamente ha distinguido al trabajo de los enseñantes en los países de la región (Rockwell, 2015 y 2018; Feldfeber, 2016).

En cuarto lugar, cabe señalar la relevancia que adquieren, en el período bajo estudio, los mecanismos de difusión y visibilidad de los resultados de las evaluaciones. De allí, los constantes intentos por modificar la legislación que regula la publicación de resultados escolares asociados a la evaluación. En buena medida, estos ensayos guardan relación con la intención de avanzar hacia la elaboración de rankings entre escuelas, de manera de otorgar mayor libertad a las familias para elegir el tipo de educación y alentar la competencia entre los establecimientos. En esta línea, el uso político y mediático de los resultados de estas pruebas, ha sido una cuestión analizada por distintos estudios que han puesto en evidencia su poder de influencia en las representaciones $\mathrm{y}$ en las valoraciones que las familias, docentes, directivos y sociedad en general hacen del sistema y de sí mismos (Rodrigo, 2016 y 2019).

Finalmente, las propuestas de evaluación y las acciones que las mismas suscitan pueden también leerse como expresiones del proceso de privatización y mercantilización de la educación que investigaciones recientes han evidenciado en países como Argentina (Feldfeber et al., 2018). Por un lado, porque es en torno a los programas de evaluación donde se trasladan y expresan los modos de gestión del sector privado vinculados al paradigma de la Nueva Gestión Pública (Falabella, 2015; Verger y Normand, 2015). Por otro lado, porque es en este tipo de programas, sobre todo en el procesamiento y en el análisis de los datos, donde participa activamente el sector privado reemplazando funciones que históricamente desempeñaba el Estado. A modo de ejemplo, bajo el gobierno de la Alianza Cambiemos, el Ministerio de 
Educación de la Nación entabló distintos convenios con fundaciones y ONG para procesar la información provista por las evaluaciones, ya sean nacionales como internacionales. Estos convenios, definidos por lo general por el poder ejecutivo, expresan procesos de terciarización de funciones que históricamente correspondían al Estado nacional (Feldfeber et al. 2018; Castellani, 2019).

De lo dicho hasta aquí, se desprende que las acciones destinadas a la evaluación del sistema educativo argentino, en el período bajo estudio, expresan modos particulares de regulación de la educación. Si la configuración y el funcionamiento de las escuelas del siglo XX fue objeto de una regulación de tipo burocrático-profesional, en las últimas décadas han tomado impulso regulaciones de tipo posburocrático (Barroso, 2017). Este modo de regulación ya no se distingue, necesariamente, por la legitimidad de la racionalidad y de la ley, sino por la valorización de los resultados y por la búsqueda de la eficacia (Maroy, 2006), dando lugar a una racionalidad de tipo instrumental. De allí que, dentro de este régimen de performatividad (Ball, 2003; Grinberg, 2008), lo que importa son los resultados tangibles, medibles y justificables. En efecto, en el actual modo de regulación el control a priori, por las normas, es sustituido por el control a posteriori, por los resultados. Se asiste así, al desarrollo de la regulación por los instrumentos (Barrosos, 2017), entre los cuales destaca la evaluación.

A su vez, y tal como se adelantó, este particular modo de regulación institucional posburocrático incorpora elementos vinculados al discurso de la NGP, en tanto complejo de creencias y de instrumentos asociados a la reforma del Estado y de su administración. La NGP responde a una lógica de administración que utiliza los principios gerenciales de la empresa, descentralizando las funciones para alcanzar la eficacia en su labor (Falabella, 2015; Verger y Normand, 2015). Supone así, un modo particular de administración y de gestión de lo público donde el Estado no entrega servicios de manera directa, sino que evalúa en términos de control y regulación. De hecho, a nivel mundial y en el campo de las políticas educativas en particular, la presencia de este discurso se ejerce en la definición de indicadores de calidad, en la publicación de los resultados de las escuelas en pruebas estandarizadas y en la evaluación externa de los resultados (Ball y Youdell, 2007). En el caso argentino, durante el período analizado, la preocupación por los resultados exacerbó la tendencia hacia la evaluación y estableció diversos mecanismos a partir de los cuales se buscó medir la calidad. En este escenario, el término acountability adquirió centralidad, en tanto involucra dos conceptos claves como son "rendición de cuentas" y "responsabilidad" (Parcerisa y Verger, 2016). Bajo esta lógica, las evaluaciones estandarizadas de rendimiento escolar, se consolidan como dispositivos de información y control acerca de lo que ocurre en las escuelas, y como mecanismos de responsabilización de los equipos docentes y directivos por los resultados conseguidos. En tanto sistema de acountability, se distinguen por al menos cuatro elementos: información, estándares, autoridad y consecuencias (Corvalán, 2006). Expresan así, la tendencia hacia la gestión educativa basada en los resultados y en el cumplimiento de objetivos, nutridos por la idea del movimiento de las escuelas eficaces (Falabella, 2015). 


\section{Consideraciones Finales}

El propósito del artículo ha sido presentar y poner en discusión las principales medidas que integraron el plan nacional de evaluación impulsado por el gobierno argentino de la Alianza Cambiemos durante el período 2015-2019. Al respecto, se destacó el lugar de estas propuestas en un contexto global de revitalización de la medición y estandarización de las prácticas educativas, donde los datos de las evaluaciones se convierten en discursos válidos para informar la gestión pública. Bajo lógicas tecnocráticas e instrumentales, la calidad educativa se reduce a la aplicación de pruebas estandarizadas, reafirmándose la cultura de la rendición de cuentas y reduciendo la educación a sus aspectos medibles y cuantificables. Sin duda, esta manera particular de regulación de las políticas educativas, afecta la forma de la escolaridad y complejiza la gestión de los asuntos escolares, las decisiones y acciones de los actores institucionales (Barroso, 2017). En tanto dispositivos de regulación de los procesos de enseñanza, los programas y políticas de evaluación operan dando forma a la escolaridad, a las prácticas y dinámicas cotidianas que distinguen a la vida de las escuelas y de sus actores. En esta línea, cobra relevancia la pregunta acerca de las particularidades que asume la experiencia de los dispositivos pedagógicos en los territorios en tiempos de gerenciamiento de la vida social (Grinberg, 2017). En este sentido, se vuelve necesario indagar en las maneras en que las escuelas se ven afectadas por las políticas y prácticas de evaluación, y cómo redimensionan las mismas en tanto modos centrales de regulación de la enseñanza. Esta mirada se torna clave tanto en lo que refiere a la producción de conocimiento en la materia, como en lo referido al diseño de políticas y al trabajo más local en las instituciones, donde la evaluación es una práctica constante que atraviesa y define la vida escolar.

En esta línea, es importante destacar la complejidad de las políticas de evaluación, en tanto involucran disputas simbólicas e instrumentales relacionadas con la construcción de la realidad socioeducativa (Monarca, 2015). Las evaluaciones pueden ser analizas como sistemas simbólicos que promueven la integración social en un orden arbitrario (Bourdieu y Wacquant, 2008), vinculándolas a disputas relacionadas con la percepción y la construcción del mundo social. Mirar desde este prisma a los sistemas de evaluación supone abordarlos como sistemas que distribuyen y configuran representaciones sobre el mundo social y educativo. Se los ubica así, en el terreno de lo político, del poder, de sus luchas por dar significado y dirección a lo real. Los sistemas de evaluación como realidad construida se encuentran en permanente interacción con sujetos, instituciones y contextos diversos. Por lo tanto, no sólo intervienen en la definición de identidades individuales e institucionales, sino que también son fabricados por ellas. Comprender sus sentidos y efectos exige entonces, el análisis de estas dinámicas y de sus particulares despliegues en el ámbito escolar. 


\section{Referencias}

BALL, S. Profesionalismo, gerencialismo y performatividad, Revista Educación y Pedagogía, Vol. XV, N³6, (mayo-agosto), 87-104, 2003.

BALL, J. y YOUDELL, D. La privatización encubierta en la educación pública. Instituto de Educación, Universidad de Londres. 2007.

BANCO MUNDIAL Profesores excelentes. Cómo mejorar el aprendizaje en América Latina y el Caribe. Resumen, Washington, DC, 2014

BARROSO, J. Dirección de escuelas y regulación de políticas: en busca del unicornio, en MIRANDA, E. y LAMFRI, N. (org.) La educación secundaria. Cuando la política educativa llega a la escuela. Buenos Aires, Miño y Dávila, 2017.

BOURDIEU, P. Y WACQUANT, L. Una invitación a la sociología reflexiva. Buenos Aires, Siglo XXI, 2008.

BRENER, G. y GALLI, G. Inclusión y calidad como políticas educativas de Estado o el mérito como opción única de mercado. Buenos Aires: Editorial La Crujía, Stella y la Fundación La Salle Argentina, 2016.

CASTELLANI, A. ¿Qué hay detrás de las fundaciones y ONGs educativas? Las redes de influencia público-privadas en torno a la educación argentina (2015-2018). Informe de investigación $\mathbf{N}^{\circ}$ 6, Observatorio de las Elites, CITRA, UMET-CONICET, 2019.

ESCANDELL, S. Operativos Nacionales de Evaluación: su incidencia en el proceso de toma de decisiones en el Ministerio de Educación Nacional (2010-2015). Trabajo final de Msaestría, Administración Pública, UBA, 2016.

FALABELLA, A. El mercado escolar en Chile y el surgimiento de la Nueva Gestión Pública: el tejido de la política entre la dictadura neoliberal y los gobiernos de la centroizquierda (1979 A 2009), Educ. Soc., Campinas, Vol. 36, № 132, pp. 699-722, jul.-set, 2015.

FEENEY, S. y DIKER, G. La evaluación de la calidad. Un análisis del discurso oficial, Revista del Instituto de Investigaciones en Ciencias de la Educación, Año VII, № 12, Agosto, 1998.

FELDFEBER, M. Facsímil: Algunas notas para analizar el discurso hegemónico sobre la calidad y la evaluación, en Brener, G. y Galli, G. (comps.) Inclusión y calidad como políticas educativas de Estado: o el mérito como opción única de mercado, CABA: Stella Ediciones, 2016.

FELDFEBER, M. y GLUZ, N. Las políticas educativas a partir del cambio de siglo: alcances y limites em la ampliación del derecho a la educación em la Argentina, Revista Estado y Políticas Públicas, №13, Octubre 2019, pp. 19-38, 2019.

FELDFEBER, M. y GLUZ, N. Las políticas educativas en Argentina: Herencias de los '90, contradicciones y tendencias de nuevo signo, Educ. Soc., Campinas, Vol. 32, № 115, p. 339-356, abr.-jun, 2011. 
FELDFEBER, M.; PUIGGRÓS, A.; ROBERTOSN, S. y DUHALDE, M. La privatización educativa en Argentina, CABA, CTERA, 2018. Disponible en:

https://ei-ie-al.org/sites/default/files/docs/investigacion_argentina_0.pdf

FERRER, G. Sistemas de Evaluación de Aprendizajes en América Latina Balance y

Desafíos. Santiago de Chile, Programa de Promoción de la Reforma Educativa en América Latina y el Caribe (PREAL), 2006. Disponible en:

http://www.rinace.net/bliblio/Ferrer2006.pdf

FRIEDRICH, D. S. Global microlending in education reform: Enseña por Argentina and the neoliberalization of the grassroots. Comparative Education Review, 58, n. 2, pp. 296-321, 2014.

GRINBERG, S. Dispositivos, gobierno de si-de los otros y escolaridad en las sociedades de gerenciamiento. En: MIRANDA, E. y LAMFRI, N. (org.) La educación secundaria. Cuando la política educativa llega a la escuela, Buenos Aires, Miño y Dávila, 2017.

GRINBERG, S. Educación y poder en el siglo XXI. Gubernamentalidad y pedagogía en las sociedades de gerenciamiento, Buenos Aires, Miño y Dávila, 2008.

GLUZ, N. Las políticas educativas destinadas a la atención de las desigualdades y los patrones de intervención sobre "la cuestión social" en el campo escolar. Ponencia presentada en el XIV Congreso Nacional de Ciencia Política, SAAP y UNSAM, San Martín, 17 al 20 de julio de 2019.

GVIRTZ, S.; LARRIPA, S. y OELSNER, V. Problemas técnicos y usos políticos de las evaluaciones nacionales en el sistema educativo argentino. Archivos Analíticos de Políticas Educativas, Volumen 14, № 18. Julio, 2006.

JAKOBI, A. y MARTENS, K. La influencia de la OCDE en la política educativa nacional, en X. Bonal; A. Tarabini-Castellani y A. Verger (comps.). Globalización y Educación. Textos Fundamentales, pp. 233-253, Buenos Aires, Miño y Dávila, 2007.

LEIBFRIED, S. y MARTENS, K. PISA: Internacionalización de la política educativa o ¿cómo se llega de la política nacional a la OCDE? Profesorado. Revista de Currículum y Formación del Profesorado, v. 13, n. 2, pp. 1-11, 2009.

MAROY, C. École, régulation et marché. Une comparaison de six espaces scolaires locaux en Europe, Paris, PUF, 2006.

MONARCA, H. (Coord.) Evaluaciones externas. Mecanismos para la configuración de representaciones y prácticas en educación. Buenos Aires, Miño y Dávila, 2015.

NARDOWSKY, M La evaluación educativa en la Argentina. De los operativos nacionales a los boletines escolares. Buenos Aires, Prometeo, 2002.

PASCUAL, L. y ALBERGUCCI, M. L La calidad educativa y su evaluación: viejos conceptos, nuevos significados, en BRENER, G. y GALLI, G. (comps.) Inclusión y calidad como políticas educativas de Estado: o el mérito como opción única de mercado, CABA, Stella Ediciones, 2016. 
PARCERISA, L. y VERGER, A. Rendición de cuentas y política educativa: Una revisión de la evidencia internacional y futuros retos para la investigación. Profesorado, Vol. 20, Núm. 3, 2016.

RAVELA, P. et. al. Las evaluaciones educativas que América Latina necesita. Documento de Trabajo № 40, Programa de Promoción de la Reforma Educativa en América Latina y el Caribe (PREAL). Santiago de Chile, 2008.

ROCKWELL, E. "Contradicciones de la evaluación del desempeño docente: lo que muestra la evidencia cuantitativa”. Educación, Formación e Investigación, Vol.1, N 1 , pp. 2422-5975, Abril de 2015.

ROCKWELL, E."La complejidad del trabajo docente y los retos de su evaluación: resultados internacionales y procesos nacionales de reforma educativa", Cuadernos de Educación, Año XVI, Núm. 16, Diciembre, 2018.

RODRIGO, L. “Los programas internacionales de evaluación estandarizada y el tratamiento de sus datos a nivel nacional. El caso de Argentina en el estudio PISA de la OCDE”. Foro de Educación, Vol. 17, Núm. 26, Enero-Junio, pp. 73-94, 2019.

RODRIGO, L. "Entre la formalidad de integrar la evaluación y el desinterés hacia sus datos. El caso de Argentina en las pruebas PISA de la OCDE". Revista Temas de Educación, N. 22, Vol. 1, Primer semestre, Universidad de La Serena, Chile, pp. 147165, 2016.

VERGER, A. y BONAL, X. La estrategia educativa 2020 o las limitaciones del Banco Mundial para promover el 'aprendizaje para todos, Educ. Soc., Campinas, Vol. 32, Núm. 117, pp. 911-932, 2011.

TORANZOS, L. Evaluación educativa: hacia la construcción de un espacio de aprendizaje. Propuesta Educativa, N 41, Año 23, Jun. 2014, Vol. L, pp. 9-19, 2014.

TENTI FANFANI, E. “Los docentes y la evaluación”. En IIPE-UNESCO (2003) Evaluar las Evaluaciones. Una mirada política acerca de las evaluaciones de la calidad educativa. IIPE-UNESCO, Sede Regional Buenos Aires, pp. 165-194, 2003. 